\title{
La filosofía en la Conquista de Silvio Zavala. Aportes y límites de una historia de las ideas*
}

\author{
Francisco Quijano Velasco **
}

\section{Abstract}

This article examines Silvio Zavala's studies of the political and philosophical controversies on the Conquest of America. The analysis of its content and methodology, as well as the discussions in which he participated with these works, shows how the historian took on a perspective closer to the so-called "traditional history of ideas" than the scientism or positivism with which it is commonly associated.

Herein Zavala's important contributions to the field of Spanish American political thought are recognized. However, it is stressed the limits of his methodological approach, which prevented him from making a major historiographical contribution.

Key words: History of ideas, conquest of America, colonialism, spanish american political thought.

* Agradezco a Alonso Sandoval su ayuda en el proceso de elaboración de este artículo, así como a los dictaminadores anónimos por la pertinencia de sus críticas y sugerencias.

** Doctor en Historia, investigador del Instituto de Investigaciones Históricas, UNAM; correo electrónico: fquijanov@gmail.com. 


\section{Resumen}

En este artículo se revisan los estudios de Silvio Zavala sobre los debates políticos y filosóficos en torno a la Conquista de América. Al analizar su contenido y metodología, así como las discusiones en los que participó con dichos trabajos, se muestra cómo el historiador adoptó en ellos una perspectiva más cercana a la llamada "historia tradicional de las ideas" que al cientificismo o positivismo con el que comúnmente se le asocia.

El artículo reconoce los grandes aportes de Zavala al campo de estudio sobre el pensamiento político hispanoamericano, no obstante, se señalan los límites del enfoque metodológico del que partió el autor, los cuales le impidieron llevar a cabo una transformación historiográfica de mayor calada.

Palabras clave: Historia de las ideas, conquista de América, colonialismo, pensamiento político hispanoamericano.

Qilvio Zavala es uno de los historiadores mexicanos más destacados del siglo XX. Su labor en la creación y dirección de instituciones y revistas, sus estudios pioneros que abrieron nuevos campos de investigación, su trabajo en la recopilación y publicación de fuentes documentales y sus obras de síntesis y difusión permiten reconocerlo como tal. Su aproximación a la época colonial — a su histórica jurídica, intelectual, económica o social— desde una perspectiva que algunos autores han definido como cientificista resultó en la elaboración de trabajos que al día de hoy siguen siendo material de consulta obligada por la información abundante y sistematizada que contienen. Pienso en La encomienda indiana, Las instituciones jurídicas de la Conquista de América, en sus estudios sobre el servicio personal de los indios en Nueva España y Perú o en las Fuentes para la historia del trabajo en Nueva España. ${ }^{1}$ Por ello, al valorar su labor historiográfica es común que se ponderen sus aportes en el campo de la investigación empírica y descriptiva más que en el de los grandes modelos interpretativos del pasado.

Aunque buena parte de la obra de Zavala puede asociarse con una postura cientificista - neopositivista, la han denominado algunos - con altas pretensiones de objetividad y basada en un riguroso trabajo de archivo, existen otros estudios del historiador yucateco de carácter, podríamos decir, más ensayístico, que adoptan una perspectiva reflexiva y en los que se

$1 \quad$ Zavala, La encomienda indiana; Las instituciones juridicas en las Conquista de América; El servicio personal de los indios en la Nueva España, (siete volúmenes); El servicio personal de los indios en el Perú, (siete volúmenes); Fuentes para la historia del trabajo en la Nueva España (1575-1805), (ocho volúmenes). 
hacen juicios de valor sobre el pasado. En dichos textos, la preocupación explícita por atender problemas del presente tiene un lugar más importante que la búsqueda por describir "lo que verdaderamente pasó", por parafrasear la frase célebre de Leopold Von Ranke. Este grupo de estudios es el que analizo en el presente artículo. En concreto, examino una serie de trabajos que abordan las controversias políticas que se presentaron en torno a la Conquista de América. En ellos reviso, por un lado, la forma en que Zavala describió lo que él mismo denomina la filosofía de la Conquista, así como las razones por las que consideraba relevante estudiarla. Por otro, reflexiono sobre la manera en que el autor se posicionó historiográficamente en estos trabajos y la metodología que siguió al realizarlos, la cual se acerca más a ciertas vertientes de la historia de las ideas que la perspectiva empirista o cientificista con la que se le suele identificar.

\section{LOS ESTUDIOS Y SU PERSPECTIVA}

El problema de la Conquista de América y las discusiones jurídicas y teológicas que desencadenó ocuparon un lugar central en la obra de Silvio de Zavala. Dicho tema atraviesa de manera transversal la mayor parte de sus trabajos, tanto los de carácter cientificista como sus ensayos de historia de las ideas.

El tema es tratado extensamente desde sus obras fundadoras de 1935: $\mathrm{La}$ encomienda indiana y, sobre todo, Las instituciones jurídicas en la Conquista de América. Durante las siguientes décadas Zavala continuó explorando esta problemática y publicó diversos libros sobre ella. Entre éstos destacan La filosofía política en la Conquista de América, los Ensayos sobre la colonización española en América y Por la senda hispana de la libertad. ${ }^{2}$ También se pueden contar el Ideario de Vasco de Quiroga, el Recuerdo de Bartolomé de Las Casas y su estudio sobre la Servidumbre natural y libertad cristiana según los tratadistas españoles de los siglos XVI y XVII, entre otros. ${ }^{3}$

En términos de contenido y de trabajo sobre fuentes documentales, la parte medular de estos estudios está en Las instituciones jurídicas en la Conquista de América. El análisis de distintos autores que Zavala llevó a cabo en este libro le permitió redactar, en la década de los cuarenta, un corpus de seis o siete ensayos centrados en la filosofía de la Conquista. ${ }^{4}$ Estos textos

2 Zavala, La filosofia politica en la Conquista de América; Ensayos sobre la colonización española en América; Por la senda hispana de la libertad.

3 Zavala, Ideario de Vasco de Quiroga; Recuerdo de Bartolomé de Las Casas; Servidumbre natural y libertad cristiana según los tratadistas españoles de los siglos XVI y XVII.

4 Con algunas variantes en los títulos, podríamos enlistar los siguientes: "Cristiandad e infieles"; "Servidumbre natural y libertad cristiana"; "Igualdad dieciochesca"; "Las Casas ante la doctrina de la servidumbre natural”; “Las Casas Esclavista?”; "Ideario de Vasco de 
los reeditará Zavala en diversas ocasiones a lo largo de su vida en las obras arriba mencionadas, alternando el orden de la presentación, agregando información o introduciendo nuevas reflexiones. En los trabajos que el mismo Zavala considera de difusión elimina el aparato crítico y presenta una breve introducción para destacar la relevancia contemporánea del problema. Las variantes que presentan los artículos en las distintas publicaciones no alteran su tesis central, sobre la que volveremos más adelante. Basta señalar por ahora que las investigaciones de Zavala de las discusiones en torno al proceso de colonización de América, realizadas a mediados de los treinta, adoptaron una carga más ideológica y normativa al participar en las controversias políticas de la agitada década de los cuarenta.

Quisiera detenerme un momento para tratar sobre los aspectos teóricos o metodológicos de la obra de Silvio Zavala, en particular de sus estudios sobre la filosofía en la Conquista. Por defender el carácter científico de la disciplina histórica, es decir, la viabilidad y deseo de que ésta produzca conocimiento objetivo del pasado a través del trabajo sobre fuentes documentales, Zavala suele ser descrito como un autor cientificista, empirista o neopositivista. ${ }^{5}$ Esta concepción de la historia se manifiesta en parte de su producción historiográfica $\mathrm{y}$, sobre todo, en su posicionamiento en los debates sobre la profesionalización de la historia que tuvieron lugar a mediados del siglo $\mathrm{xx}$ y que lo confrontaron con historiadores vinculados a la tradición del historicismo alemán. ${ }^{6}$

Quiroga". Algunos fueron resultado de conferencias, otros artículos publicados en libros y revistas.

5 Sobre la valoración de la obra de Zavala véase González, Silvio Zavala y el quehacer histórico en México, pp. 7-19; Matute, Herrejón Peredo, Aproximaciones a la obra de Silvio Zavala, pp. 131-135; Dougnac, La inspiración medieval allende el atlántico: Silvio Zavala (1909-2014), pp. 55-73; Vázquez, La historiografia mexicana en las décadas recientes; León-Portilla, Corrientes o tendencias en la historiografia mexicana...; Moctezuma, El camino de la historia hacia su institucionalización, pp. 47-50; Zermeño, La historiografía en México, un balance (1940-2010), pp. 1695-1699.

En una entrevista que le hizo Peter Bakewell en 1982, Zavala critica la historiografía de la segunda mitad del siglo XX, que consideraba presuntuosa y que, a su juicio, tendía a imponer al pasado modelos e ideas del presente. En contraste, defendía una forma de hacer historia que se apegara más a los documentos, reconociendo su valor intrínseco. Véase Zavala, Bakewell, y Gutiérrez Mills, "An Interview with Silvio Zavala".

6 Las décadas de los cuarenta y cincuenta fueron ricas en debates historiográficos, en gran medida como resultado del proceso de la profesionalización de la historia en México. Quienes han estudiado las discusiones de este periodo, presentes en el ámbito académico e institucional (enseñanza de la historia, libros de texto y planes de estudio) dan cuenta de dos grandes corrientes historiográficas que se enfrentaron: la historicista y la cientificista $o$ positivista. Véase Moctezuma, El camino de la historia hacia su institucionalización, pp. 47 50; Zermeño, La historiografia en México, un balance (1940-2010), pp. 1695-1699; Valero, José Gaos en México: una biografia intelectual, capítulo "12 La balsa de piedra”, pp. 343367 , entre otros. 
No obstante, como veremos más adelante, sus estudios sobre la filosofía política en la Conquista se alejan de las pretensiones de objetividad de la historiografía positivista, así como de sus objetivos meramente descriptivos. La perspectiva reflexiva adoptada por Zavala en dichos trabajos devino en argumentos normativos que buscaron incidir explícitamente en discusiones filosóficas, políticas e historiográficas de su tiempo.

Al estudiar las controversias sobre la colonización de América, Zavala procedió como un historiador de lo que hoy llamaríamos - recuperando un concepto acuñado en la década de los sesenta - "historia tradicional de las ideas". Hacia mediados del siglo xx, la mayor parte de los estudios de historia del pensamiento se realizaba bajo esta perspectiva. Ésta, sin embargo, partía de una concepción de la disciplina histórica diferente a la que subyacía en las corrientes cientificistas e historicistas. La llamada historia tradicional de las ideas no buscaba restituir la historicidad de la actividad intelectual del pasado, ni tampoco explicar el sentido o función que tenían las ideas dentro de su contexto histórico. Su objetivo, en cambio, era mostrar la dimensión trascendental del pensamiento filosófico. Por ello, quienes adoptaron esta perspectiva se interesaron en analizar el tratamiento que los pensadores del pasado dieron a los "grandes problemas" de la historia de la filosofía, abstrayéndolos de su contexto lingüístico y sociopolítico. Otra de sus características fue el interés por demostrar la relación de continuidad, normalmente a partir de cadenas de influencia, que existía entre los pensadores de distintos momentos de la historia. Esto, como lo hizo notar en su momento Quentin Skinner, producía lecturas teleológicas del pasado que valoraban a los autores no en función de su contexto, sino de lo que había sucedido después, por ejemplo, atribuyéndoles haber fundado una doctrina o tradición intelectual o haberse "adelantado a su época"?

Los estudios de Zavala sobre el pensamiento en la Conquista reproducen algunas de las características de la historia de las ideas que se acaban de describir. Es interesante, por ejemplo, la poca atención que presta el autor al contexto sociopolítico al abordar el problema de la Conquista y su legitimación, sobre todo considerando que Zavala fue un especialista de la historia política y social de Nueva España. Esto lo hace explícito el mismo autor al apuntar que en dichos estudios no le interesan los tiempos marcados por los acontecimientos sino los fenómenos jurídicos e intelectuales de larga duración. ${ }^{8}$ Como veremos enseguida, la intención del autor al abordar estos temas fue dar cuenta de la existencia de una larga tradición intelectual

7 Quentin Skinner denominó "mitologías” a la producción de la historia tradicional de las ideas que adoptaba las características mencionadas. Sobre esto, véase Skinner, Visions of Politics I, Regarding Method.

$8 \quad$ Zavala, Ensayos sobre la colonización española en América, p. 14. 
hispanoamericana que corría del temprano siglo XVI al siglo xx y, junto con ello, mostrar la riqueza y vigencia de las ideas desarrolladas dentro de dicha tradición.

\section{El ARgumento}

Dejemos por ahora las cuestiones metodológicas y formales de los estudios de Zavala sobre la filosofía de la Conquista para analizar qué dice el historiador al tratar sobre estos temas y qué sentido tienen sus argumentos. Para responder estas preguntas es conveniente, en primer lugar, situar los escritos de Zavala en el contexto polémico de su tiempo, es decir, entender qué y frente a quiénes discutió en las obras mencionadas. Sobre esto, es posible identificar, cuando menos, dos espacios de controversia interrelacionados en los que el autor buscó incidir: uno eminentemente historiográfico y el otro de carácter político.

El primero de ellos tiene que ver con la forma en que era valorado el periodo colonial dentro de la historiografía de su época; el problema del lugar que ocupaban - y debían ocupar - los siglos XVI, XVII y XVIII en el recuento de la historia hispanoamericana, particularmente la historia de su pensamiento. Zavala criticó las lecturas de la historiografía de corte liberal que desde el siglo XIX tendían a considerar a la Colonia como un lastre de las naciones hispanoamericanas que no merecía ser estudiado. Frente a ellas, el autor reivindicaba la significación y trascendencia de la Modernidad temprana de Hispanoamérica y la importancia de estudiarla en toda su complejidad. Dice Zavala al referirse a quienes elaboraban ese tipo de historia (sin especificar puntualmente a quién):

[Ellos] creyeron que [la etapa de la colonización española] constituía un largo periodo de letargo, de interés remoto, separado definitivamente de la historia de los pueblos hispanoamericanos por la declaración de independencia de España. Se describían los trescientos años coloniales como un preludio del verdadero drama de nuestra historia, iniciado por los libertadores del siglo XIX. ${ }^{9}$

Contrario a esta opinión, Zavala consideraba al siglo XVI como el momento en que había irrumpido la Modernidad en América Latina. El periodo colonial, además de haber sido determinante en el desarrollo de los pueblos hispanoamericanos, se trataba, a su juicio, de una etapa rica en ideas y tradiciones filosóficas que merecían ser analizadas por sí mismas. El autor veía en la colonización un proceso que posibilitó la aparición de un pensamiento de carácter universal, cuyos valores podían ser recuperados para 
reflexionar sobre el presente. Al finalizar sus ensayos sobre La colonización española en América apunta lo siguiente:

En conclusión, yo me daría por satisfecho si, a lo largo de estos ensayos, hubiera logrado mostrar las razones que existen para creer que la colonización española en América contiene una rica ideología social y una experiencia sustanciosa. Esa colonización puede estudiarse con provecho si se deja de lado los prejuicios que nos han acostumbrado a ver en ella una masa histórica inerte y de escasa fuerza constructiva. ${ }^{10}$

Zavala, en este punto, está atendiendo un problema historiográfico. No obstante, su llamado a revalorar, de manera positiva, la historia colonial tiene implicaciones éticas y políticas para el contexto en el que lo hace. Estas implicaciones son las que dan forma al segundo espacio en el que Zavala está interviniendo con sus textos. Éste es más "cercano" en términos del presente del autor y es definido por él mismo como "el problema colonial moderno". El enfoque que adopta Zavala ante esta problemática es histórico en tanto que acude a la historia como punto de partida para unas reflexiones de carácter ético o moral. La propuesta de Zavala es establecer un paralelismo entre los problemas del colonialismo debatidos en el siglo XVI con los que se presentaron en los siglos XIX y XX.

La cuestión medular del colonialismo moderno, que para Zavala atraviesa de manera transversal todo su desarrollo histórico, es la pregunta sobre las razones para justificar o denunciar el dominio de un pueblo sobre otro. En este sentido, Zavala se interesa más por los fundamentos morales o políticos que legitimaban al colonialismo que por los elementos económicos que habrían funcionado como su motor. ${ }^{11}$ El historiador distingue entonces una tradición que dominó las discusiones sobre el imperialismo y el colonialismo a lo largo de la Modernidad, cuyo argumento central era el deber que tenían los pueblos avanzados de civilizar a las naciones inferiores. Este deber podía partir de principios religiosos (siglo XVI), de la idea del progreso (siglo XIX y primera mitad del xx) o de una suerte de fraternidad u obligación moral para restituir la justicia (mediados del siglo xx). El autor de La filosofía política en la Conquista de América presenta rápidamente algunos ejemplos de personajes o momentos en donde se manifestó esta tradición, desde la Utopía de Tomás Moro hasta la creación del Consejo de Tutela de la ONU (el Consejo de Administración Fiduciaria) establecido en 1945, poco antes de que redactara dicho texto.

Ibid., p. 159.

Me refiero aquí a sus trabajos sobre la filosofía de la Conquista. Zavala atiende en otros estudios las dimensiones socioeconómicas del colonialismo americano. Entre éstos destaca su trabajo ya referido sobre la encomienda indiana y su libro Los intereses particulares en la conquista de la Nueva España. 
La intención de Zavala al describir esta tradición es mostrar cómo las discusiones sobre la descolonización de su presente podrían enriquecerse con los argumentos utilizados en los debates sobre la Conquista de las Indias del siglo XVI. ${ }^{12}$ Zavala, así, introduce un argumento con el que se posiciona, al mismo tiempo, en las controversias historiográfica y política mencionadas: en el mundo hispanoamericano existe una larga tradición de pensamiento liberal; y ésta — si bien tiene antecedentes en fuentes clásicas y medievales - tuvo su origen en las discusiones sobre la colonización de las Indias. Así comienza $L a$ filosofía política en la Conquista de América:

Se ha pensado que la idea de libertad nace en Hispanoamérica con la victoria que obtuvieron los partidarios de la Independencia sobre los defensores del sombrío pasado colonial. Sin embargo, creemos descubrir las raíces de una inclinación favorable a esa prerrogativa humana desde que ocurre el primer contacto del Nuevo Mundo con la cultura de Europa.

Si esta suposición es correcta, y si las pruebas en que descansa resisten a la crítica y al tiempo, se podrá extender la historia de nuestro liberalismo a campos más amplios y a épocas más remotas. Con ello, acaso, sus raíces aparecerán dotadas de mayor penetración y firmeza. ${ }^{13}$

Zavala describe en las obras en cuestión el origen y el desarrollo de esta tradición liberal durante la temprana Modernidad. Para ello, analiza el pensamiento de personajes como Juan López de Palacios Rubios, Matías de Paz, Francisco de Vitoria, Juan Ginés de Sepúlveda, Bartolomé de Las Casas, Vasco de Quiroga o José de Acosta. ${ }^{14}$ A diferencia de otros actores que tomaron parte en este proceso y que no cuestionaron las bases jurídicas o morales de la Conquista, como los conquistadores y colonos, todos estos pensadores se preguntaron por los términos en que debía llevarse a cabo. Por ello, Zavala los agrupa dentro de un conjunto que denomina paternalista o proteccionista en el que, pese a su diversidad interna, reconoce los elementos constitutivos de lo que concibe como liberalismo hispanoamericano. La estrategia que sigue al revisar estos autores es describir los argumentos que utilizaron para justificar o denunciar la colonización, poniendo especial atención al problema de la libertad.

12 Para Zavala no resulta difícil establecer un paralelismo entre el siglo XVI y el siglo XX: "porque no pocas veces han surgido las circunstancias que rodean a la expansión de naciones poderosas y al gobierno colonial de pueblos. Esto nos autoriza a interpretar la Conquista española de América como un antecedente valioso de la presente experiencia internacional y política, aunque no sean idénticas la terminología ni la individualidad histórica en cada caso", Zavala, Por la senda ..., p. 16.

13 Zavala, La filosofia política en la Conquista... p. 15.

14 En menor grado, remite también a otros pensadores de los siglos XVII y XVIII, como Juan de Palafox, Juana Inés de la Cruz, Francisco Xavier Clavijero o Servando Teresa de Mier. 
Ahora bien, las diferencias que encuentra Zavala en las ideas de los autores referidos le permiten distinguir dos grandes vertientes dentro de este grupo: por un lado, la renacentista imperial y, por otro, la libertaria cristiana. En sus ensayos ubica a los autores que participaron en las discusiones sobre la Conquista de América dentro de una u otra vertiente. Así, por ejemplo, Palacios Rubios o Matías de Paz pertenecen a la vertiente imperial, mientras que Francisco de Vitoria o Vasco de Quiroga, a la liberal cristiana. Pero la parte más amplia de su análisis se centra en dos personajes que, a su juicio, condensarían lo sustancial de cada una de estas tradiciones: Ginés de Sepúlveda y Bartolomé de Las Casas.

Zavala describe cómo Sepúlveda recuperó la teoría clásica de la servidumbre natural planteada por Aristóteles, así como su concepto de barbarie, para analizar y valorar a los indígenas americanos. Para el autor, es a partir de ellos que el humanista defendió la legitimidad del domino español sobre América dentro de un proyecto colonizador y civilizatorio. Zavala vincula así el pensamiento de Sepúlveda con una tradición renacentista que justificaba la jerarquización de las naciones de acuerdo con su desarrollo. Las Casas, por su parte, explica Zavala, a partir de una filosofía profundamente cristiana defendió la igualdad y libertad de todos los hombres de la tierra. Con ello, el dominico atacó desde su base la teoría de la servidumbre natural expuesta por su contrincante. Para Zavala, el argumento central de Las Casas, del que se deriva toda su teoría política, es que Dios creó a todos los seres humanos con la misma naturaleza racional. Por ello, no podían ni debían ser sometidos a un gobierno extranjero sin su consentimiento. Si Sepúlveda era concebido como un humanista del renacimiento, Las Casas aparece aquí como un pensador católico, heredero de la filosofía cristiana medieval. ${ }^{15}$ Cabe señalar que Zavala no se detiene a analizar de manera integral la filosofía política de Las Casas ni de Sepúlveda. No revisa, por ejemplo, la postura que estos autores guardaron ante temas como el origen o la legitimidad del poder político. Aunque en algún pasaje menciona que Las Casas era partidario de la teoría de la soberanía popular, reduce la explicación sobre su crítica a la conquista a la dimensión religiosa de su pensamiento. No obstante, como veremos más adelante, el autor regresará a este último punto al revisar a los autores de la Ilustración.

Al describir ambas vertientes del liberalismo hispanoamericano del siglo XVI, Zavala no se abstiene de realizar juicios de valor. A diferencia de lo que algún lector podría esperar, el historiador no concibe a las posturas renacentista imperial y a la libertaria cristiana como reprobable y aceptable, respectivamente, sino que en ambas ve aspectos positivos que deben ser recuperados. Para Zavala, las dos tradiciones hispanoamericanas aportan 
ideas y experiencias sustanciosas para comprender y pensar el problema del colonialismo en el contexto de la posguerra.

La primera, pese a su carácter imperial, resulta útil, ética y analíticamente, para discutir al colonialismo como fenómeno histórico. En primer lugar, señala el autor, esta vertiente adopta una lectura "realista" de la política internacional al asumir como punto de partida - y como un problema a resolver - las diferencias en el desarrollo de los pueblos, algo que, a su juicio, no contemplan los proyectos humanitarios de la tradición liberal cristiana. Para Zavala, esta lectura da cuenta de la historicidad de las naciones y sitúa a la filosofía política en una dimensión más pragmática. Pero su relevancia radica, sobre todo, en que se presenta como un proyecto imperialista moralizante y civilizador. Con ello, el historiador establece una distinción con otras formas de imperialismo vinculadas, sobre todo, a las naciones del norte de Europa. Zavala considera que el imperialismo renacentista español fue erróneamente considerado un antecedente directo del esclavismo y el racismo del siglo XIX y del nazismo de su tiempo. Para él, existe una ruptura entre dicha tradición y las que desarrollaron teorías deterministas de siglos posteriores, por lo que estas dos formas de imperialismo, que suelen considerarse como una misma, correrían de manera paralela. La ruptura radica en que el parámetro de diferenciación del primero no se ubicaba en la raza, sino "en la racionalidad" o en el grado de civilización de los pueblos, siempre perfectible. Y, sobre todo, en que el último fin de aquel colonialismo no era el beneficio del colonizador sino del colonizado.

Para Zavala, todo esto no se reconoce al estudiar a autores como Sepúlveda, por lo que su pensamiento ha sido comúnmente mal interpretado. ${ }^{16}$ Al reivindicar el valor de esta tradición Zavala no defiende el colonialismo, aunque simpatiza con la idea de que existe una obligación moral de los pueblos civilizados de auxiliar a aquéllos que no han alcanzado un grado razonable de desarrollo.

No obstante, Zavala encuentra en la segunda vertiente de esta tradición, la libertaria cristiana, una propuesta más rica y moralmente más celebrable. El historiador ve en ella una modernidad ética y política anterior a la del siglo XVIII, en la que se reconoció la dignidad, igualdad y libertad de todos los seres humanos. Con ello vincula el origen de estos principios políticos, centrales de la Modernidad, no a la Ilustración o a lo que hoy conocemos como liberalismo, sino a una tradición escolástica, católica e hispanoamericana. Esta modernidad fue distinta a la de Las Luces, pero no por ello menos relevante. La dimensión religiosa, que puso al centro de su "liberalismo" el servicio al prójimo y la generosidad hacia el desfavorecido, distingue para 
Zavala a esta vertiente que contribuyó "a mejorar el destino de los hombres pertenecientes a culturas distintas de la europea". ${ }^{17}$

Sostener que existe una rica tradición liberal en Hispanoamérica, tan rica que incluye dentro en sí expresiones del humanismo renacentista imperial y del cristianismo escolástico de raíces medievales, le permite a Zavala demostrar que la Modernidad temprana en el mundo hispano, lejos de ser inerte, fue un periodo de gran relevancia y riqueza intelectual en donde es posible encontrar el origen de los principios y valores políticos más preciados del mundo contemporáneo. Con ello, Zavala reubica al pensamiento católico español como un elemento clave en la historia de Occidente. En este sentido, podríamos situar al autor de La filosofía de la Conquista dentro de la vertiente americanista del regeneracionismo español que tuvo en su maestro, Rafael Altamira y Crevea, a uno de sus más importantes representantes. En el prólogo de dicho libro, el mismo Altamira reconoce los aportes de Zavala dentro del proyecto regeneracionista:

La originalidad de Zavala en este libro consiste en haber ahondado y aumentado la historia de lo que propiamente debemos llamar nuestro liberalismo (en el sentido de tolerancia y del respeto a la persona humana, que es lo fundamental en él) con relación al problema de los indígenas americanos. Por esa aportación científica le debemos la gratitud los españoles de hoy, en primer lugar; y tras estos, todos los historiógrafos que buscan, ante todo, la verdad de las realidades humanas. ${ }^{18}$

De esta forma, hacia mediados del siglo $\mathrm{xx}$, encontramos a Silvio Zavala trabajando en la renovación de la historiografía que anhelaba el regeneracionismo español. El historiador dedicó buena parte de su producción de dichos años para criticar la forma en que era concebido el pasado hispanoamericano de la temprana Modernidad y apostar, en cambio, por una nueva lectura que reconociera su trascendencia y significación. Con ello, buscaba reconciliar a los españoles y latinoamericanos con un pasado que era visto como una carga para que, una vez liberados, descubrieran en él su potencial para pensar los problemas de su presente. ${ }^{19}$

Ibid., pp. 73-110.

Ibid., pp. 10-11.

Señala Zavala al concluir La filosofia política en la Conquista: "El mensaje ideológico que se desprende de este ensayo podría resumirse en las proposiciones siguientes: La libertad es más antigua entre nosotros de lo que comúnmente se ha creído. El Cristianismo no llega al Nuevo Mundo desprovisto de fermentos favorables a la libertad humana, aunque después haya podido desviarse por otros caminos. Quienes desde la época de la contienda por la Independencia vienen defendiendo la concepción liberal de la vida no tienen que renegar del pasado hispanoamericano en su conjunto, pues contiene valores capaces de suministrar apoyo y estímulo a esa misma defensa”, p. 145. 
Como vemos, los trabajos sobre la filosofía de la Conquista de Silvio Zavala estaban lejos de los objetivos y presupuestos metodológicos de la historiografía positivista con la que se suele identificar al autor. Las pretensiones cientificistas que buscaban despojar a la disciplina histórica de su dimensión política y reflexiva, que pudieron haberse reflejado en el programa de Zavala para la profesionalización de la historia, no tuvieron lugar en su proyecto de renovación de la historia de las ideas hispanoamericanas. Este, en cambio, fue un proyecto ideológico de reivindicación del pensamiento hispánico, en sus vertientes católica e imperial.

\section{LÍMITES Y APORTES DE SU LECTURA}

El interés de Zavala en demostrar la existencia de una tradición liberal hispanoamericana de larga duración impidió que el historiador explicara y valorara, en su propio contexto, algunos aspectos medulares del pensamiento de los autores analizados. Quizá, donde esto se manifiesta de forma más clara es en el análisis que hace del impacto que tuvo Las Casas entre ciertos autores de la Ilustración. Regresemos a los textos de Zavala para observar cómo aborda esta cuestión.

Tras la revisión de los debates que se presentaron sobre la Conquista en el siglo XVI, Zavala pasa de largo por los autores del siglo XVII — a quienes les dedica apenas unas páginas - para volver a detener su mirada en el pensamiento de finales del siglo XVIII e inicios del XIX. Si bien menciona a algunos escritores hispanoamericanos de este periodo - como Clavijero, Márquez o Eguiara y Eguren - focaliza su atención en Europa y en la manera en que algunos filósofos ilustrados, particularmente franceses, remitieron a América en sus discusiones sobre la igualdad y la libertad. ${ }^{20}$ El pensamiento de Bartolomé de Las Casas es, nuevamente, un tema central en esta parte de su análisis pues, para Zavala, fueron las ideas del obispo de Chiapas las que más eco hicieron entre los pensadores europeos y americanos de la Ilustración.

Zavala advierte en sus textos diferencias entre el pensamiento de Las Casas y el de algunos pensadores del siglo XVIII. La principal tiene que ver con la dimensión eminentemente religiosa del primero y el elemento secularizador que caracterizó la filosofía de los segundos. Además, señala explícitamente que no observa una relación de influencia directa entre Las Casas y autores, por

20 Esto lo trata principalmente en el capítulo "La igualdad dieciochesca" que aparece tanto en La filosofia política en la Conquista..., pp. 111-143, como en Por la senda... pp. 56-67. Zavala cuenta además con un libro en el que trata de forma más extensa y profunda dicho problema, un trabajo que podría contarse entre sus obras eruditas: Zavala, América en el espiritu francés del siglo XVIII. 
ejemplo, como Jean Jaques Rousseau o Nicolas de Condorcet. No obstante, encuentra en Henri Grégoire a un personaje en el que se puede documentar la impronta lascasiana en la Ilustración. Esto es muy importante, pues le permite sostener que el liberalismo hispanoamericano —originado en la filosofía de la Conquista - se encontraba vigente en el Siglo de las Luces y mostrar así su trascendencia histórica. Detengámonos un momento para analizar esta cuestión. ${ }^{21}$

El fenómeno concreto que revisa Zavala es una discusión que se presentó en Francia a inicios del siglo XIX en torno a si Las Casas era o no un defensor de la esclavitud negra. Ésta tuvo lugar dentro de una polémica más amplia, suscitada a raíz de la Revolución Francesa sobre los derechos que debían tener los esclavos negros y la posibilidad de reconocer su ciudadanía. Dentro de esta discusión, para Zavala, el abate Grégoire retomó y defendió la causa libertaria del obispo de Chiapas.

La discusión de inicios del siglo XIX sobre el posible esclavismo lascasiano se disparó, explica Zavala, por la noticia que se tuvo de una petición que hizo Bartolomé de Las Casas de introducir esclavos negros a América como una forma de remediar la devastación de la población indígena. Esta situación puso en debate no sólo si Las Casas había sido un apologista de la esclavitud, sino también si a él se le debía la llegada de los esclavos africanos al continente americano. Como el mismo Zavala advierte, desde el punto de vista "documental" — así lo llama - analizar esta polémica a mediados del siglo Xx no tenía mucho sentido, pues para entonces se conocía ya el fragmento de la Historia de las Indias (que los autores ilustrados no tuvieron a su disposición) en donde el mismo Las Casas aceptaba haber hecho esa solicitud, pero al mismo tiempo se arrepentía al darse cuenta que la esclavitud de los negros era tan injusta como la de los indios. ${ }^{22}$

Superada la duda sobre lo que "realmente sucedió", Zavala se interesa en mostrar cómo a raíz de esta polémica los ilustrados entraron en contacto con el pensamiento lascasiano y cómo algunos de ellos, en particular Henri Grégoire, incorporó elementos de la tradición cristiana del siglo XVI a la filosofía de las Luces. Al tratar este asunto Zavala utiliza una estrategia que, en principio, pareciera mostrar la lectura anacrónica que Grégoire hizo de Las Casas. Dice Zavala que el abate:

[...] ponía en boca de este religioso — defensor del amor a la "humanidad" y de la igualdad de derechos - discursos propios de un ciudadano ilustrado de la época de la revolución francesa. Por ejemplo, que lo que importa a todos, exige el consentimiento de todos; que la prescripción de la libertad es inadmisible; que la

21 Véase La filosofía politica en la Conquista..., pp. 129-138, y Por la senda..., pp. 61-67. También lo trata en un pequeño artículo titulado: “LLas Casas esclavista?, publicado en Recuerdo de Bartolomé de Las Casas, pp. 49-57 y Por la senda..., pp. 95-100.

22 Dicho pasaje aparece en Bartolomé de Las Casas, Historia de las Indias, t. III, pp. 275-276. 
forma del estado político debe ser determinada por la voluntad del pueblo, porque él es la causa eficiente del gobierno, y que no se le puede imponer [al pueblo] carga alguna sin su consentimiento. Además, Las Casas aparece sosteniendo que la libertad es el mayor de los bienes y que, siendo todas las naciones libres, el quererlas sujetar bajo pretexto de que no son cristianas es un atentado contra los derechos natural y divino, y quien abusa de su autoridad es indigno de ejercerla y no se debe obedecer a ningún tirano [...].

Y concluye explicando que:

La libertad cristiana atrajo a la nueva filosofía [de la Ilustración], y ésta, para recibir la herencia, hubo previamente de retocar el cuadro histórico con fuertes pinceladas. Las diferencias se perdieron en la sombra y las semejanzas pasaron a primer plano. Pero Gregorio no era un impostor. Su discurso se apoyaba en pasajes auténticos de Las Casas... ${ }^{23}$

Efectivamente, todas las ideas de Las Casas expuestas por Zavala en boca de Grégoire son citas casi literales de pasajes de la obra del dominico. No obstante, el autor no explica cómo fue posible que Las Casas hubiera formulado esas proposiciones que parecían ser propias del pensamiento ilustrado. Como vimos, para Zavala la filosofía política de Las Casas estuvo caracterizada por su dimensión profundamente religiosa. Los fundamentos que la sostenían se reducían a dos grandes preceptos del cristianismo: la idea de que Dios había creado a todos los hombres con las mismas características y la obligación que éstos tenían de trabajar en servicio a los demás. De ahí que el liberalismo cristiano del siglo XVI fuera un humanismo paternalista y proteccionista. Esta lectura del pensamiento lascasiano impide acomodar en su contexto los principios constitucionalistas o republicanos que enlista en la cita del Abate. Zavala resuelve el asunto presentando estos presupuestos de Las Casas como los de un autor, en sus palabras, "adelantado a las luces de su siglo"; como "discursos propios de un ciudadano ilustrado de la época de la revolución francesa". ${ }^{24}$

¿Cómo explicar que Zavala no analizara estos argumentos de Las Casas en el contexto en el que fueron expuestos y no en función de lo que pudieron o no provocar en la Ilustración? Pareciera que para el autor el pensamiento de Las Casas tuviera dos dimensiones: una propia del siglo XVI, católica y paternalista; y la otra, "adelantada a su época", secular y republicana. Por ello, esta última sólo pudo trascender cuando el ambiente intelectual estuvo listo para recibirla. El objetivo de Zavala, como se mencionó, era mostrar al liberalismo hispano como una corriente intelectual que se desarrolló desde las discusiones sobre la Conquista hasta el siglo xx. Por ello, la lectura que 
hace del pensamiento de Las Casas no daña su tesis central. Al contrario, el episodio mencionado le permite documentar su argumento: el pensamiento liberal español, cuyo origen está en las discusiones de la Conquista de las Indias, emergió nuevamente en el periodo de las Revoluciones Atlánticas en donde fue reinterpretado para dar forma al último liberalismo, ilustrado y secular, que es el que ha llegado a nuestros días.

Así, desde la historia de las ideas Zavala establece una ruptura con la narrativa de la historiografía liberal, dominante en su época y aún con fuerte presencia en nuestros días. Para esta narrativa existe un quiebre dramático entre las tradiciones intelectuales del llamado Antiguo régimen y aquéllas que posibilitaron la emergencia de la Modernidad. Zavala, en cambio, ubica el origen de esta Modernidad en el ámbito hispánico más de doscientos años atrás $\mathrm{y}$, con ello, reconoce el papel que tuvo el pensamiento del siglo XVI en la configuración de algunos de los presupuestos políticos más importantes del mundo contemporáneo. No obstante, al limitar la valoración de Las Casas y de otros autores que participaron en las discusiones sobre la Conquista a un carácter paternalista y religioso, Zavala no logra eludir por completo los axiomas de esa misma historiografía que denuncia en sus trabajos.

Veinte años después de que se publicara La filosofía politica en la Conquista de América, en Inglaterra, un grupo de historiadores partieron de un diagnóstico y una crítica similar a los que había hecho Zavala a la historiografía liberal, aunque en este caso para analizar no a Hispanoamérica, sino al ámbito anglófono. Al hacerlo, y al presentar una respuesta distinta a la que brindó el yucateco, estos historiadores provocaron una revolución en la historiografía del pensamiento político moderno. Me refiero, como algunos habrán adelantado, a la llamada Escuela de Cambridge, en particular a sus dos representantes más importantes: John Pocock y Quentin Skinner. A grandes rasgos, dichos autores mostraron cómo la revolución inglesa de mediados del XVII y las revoluciones atlánticas del XVIII no estuvieron fundadas, principalmente, sobre una ideología liberal, sino sobre los presupuestos de una tradición intelectual más antigua y extendida en Europa: el republicanismo clásico; y que esta última no fue sólo un simple antecedente del liberalismo decimonónico, sino que ambas se presentaron como tradiciones, en muchos aspectos, antagónicas. ${ }^{25}$ En paralelo a sus estudios históricos, dichos pensadores desplegaron una crítica a la forma más generalizada en que procedía la historia de las ideas y propusieron nuevos enfoques teóricos y metodológicos que contribuyeron de manera decisiva a la transformación de la disciplina. ${ }^{26}$

Al remitir a la Escuela de Cambridge no busco explicar los límites del pensamiento de Zavala a partir de lo que hicieron años después otros

25 Pocock, El momento maquiavélico. El pensamiento politico florentino y la tradición republicana Atlántica; Skinner, Los fundamentos del pensamiento político moderno.

26 Véase Skinner, Visions of Politics...; y Pocock, Political Thought and History. 
historiadores. Me interesa, en cambio, mostrar el potencial que tiene - dentro del ámbito hispanoamericano - la crítica que hizo a la historiografía liberal al retomarse desde una historia intelectual que se distancie de los presupuestos de la llamada historia de las ideas. Una historia que busque, antes que otra cosa, dar cuenta de la historicidad del pensamiento y de sus condiciones de posibilidad. ${ }^{27}$

Estudiar el pensamiento de la Conquista desde una perspectiva así nos permite ver detrás de los argumentos empleados por autores como Las Casas una teoría mucho más compleja que la denominada por Zavala como libertaria cristiana. Ésta, efectivamente, defendía el derecho a la libertad de todos los hombres, pero además, partiendo de una concepción popular del origen del poder, establecía mecanismos para la limitación de la autoridad de los gobernantes mediante la voluntad del pueblo y concebía la participación de los ciudadanos en el gobierno como condición de la libertad. Asimismo, nos muestra cómo el uso de los principios expuestos por Henri Grégoire no fue excepcional en la Monarquía hispánica del siglo XVI. Las Casas no

27 Aunque gran parte de las herramientas metodológicas de la historia intelectual se desarrollaron en el último tercio del siglo $\mathrm{XX}$, desde mediados de dicho siglo encontramos argumentos que apuntan hacia la necesidad de estudiar la dimensión histórica de las ideas. Éste era uno de los objetivos que buscaba el historicismo mexicano defendido por autores como José Gaos o Edmundo O’Gorman. Así, por ejemplo, en un artículo de 1941 titulado "Sobre la naturaleza bestial del indio americano" éste último analizó temas y fuentes similares a los que Zavala abordó en sus estudios sobre la filosofía de la Conquista. Partiendo también de una pregunta trascendental (¿qué es lo humano?) O’Gorman analiza los debates que se presentaron en el siglo XVI, particularmente el de Las Casas y Sepúlveda, no para demostrar la continuidad de una tradición intelectual que vincule el pensamiento del siglo XVI con el del XX, ni tampoco para revalorar, de manera positiva, sus ideas. Lo hace, en cambio, para explicar la singularidad del pensamiento del pasado: ¿qué era lo que los autores del pasado "verdaderamente" dijeron y cómo fue posible que lo hicieran? Ahí radica para O'Gorman la utilidad de acercarse a la historia de la filosofía: entender la dimensión histórica y contingente del pensamiento.

Zavala expresa una preocupación similar en la entrevista de Peter Bakewell citada anteriormente. En ella sostiene que: "I never gave up the notion that in spite of the essential unity of men through time, however, generations are distinguished one from another by the conditions in which they live, and that diversity offers a rationale and an attraction for the historical study of generations and societies of the past. Only when the historian erects a solid bridge between his own life and those lives consumed by time is he really accomplishing his mission [...] In conclusion, I should say that in the last few years my appreciation for the documents of the past and for their intrinsic value has again gained utmost importance for me. My latest works reflect this and I would wish that all of my effort as a historian would be seen in the following terms: he studied the documents of the colonization of the New World and he left signs of having appreciated them and of having learned something of the life they hid in their pages. That is to say, a posthumous justification of the historian based on the sources he used and the way in which he handled them", Zavala, Bakewell, y Gutiérrez Mills, "An Interview with Silvio Zavala", p. 564. 
recurrió a ellos por ser un personaje adelantado a su tiempo, sino porque formaban parte de los lenguajes y presupuestos políticos presentes en el ámbito hispanoamericano en el siglo XVI. El corpus de fuentes que estaban a disposición de los autores que elaboraron la filosofía de la Conquista que incluía autoridades clásicas, medievales y renacentistas - permitió a Las Casas y a otros pensadores echar mano de esos argumentos. Con ellos no sólo criticaron los abusos del imperialismo, sino que desafiaron desde sus bases a las tradiciones teocráticas y absolutistas sobre las que éste descansaba. ${ }^{28}$

Quisiera concluir señalando que a más de 70 años de la publicación de La filosofía política en la Conquista de América, el llamado a revalorar la historia del pensamiento político de la época colonial, reconociendo su riqueza y diversidad, sigue teniendo vigencia. Habría que atender este llamado y tomarnos en serio la tarea de explicar el pensamiento hispanoamericano del siglo XVI, no en función de lo que pudo o no provocar siglos después, sino en lo que pudo o no provocar en su momento. Esto, me parece, puede contribuir a consolidar la renovación historiográfica sobre el pensamiento político hispanoamericano de la Modernidad temprana que Silvio Zavala inició con los trabajos que aquí hemos analizado.

\section{Bibliografía}

Dougnac Rodríguez, Antonio, "La inspiración medieval allende el atlántico: Silvio Zavala (1909-2014)", Revista de Dret Històric Català, Societat Catalana d'Estudis Jurídics, vol. 15, 2016, pp. 55-73.

González y González, Luis, "Silvio Zavala y el quehacer histórico en México", Historia Mexicana, vol. 39, núm. 1, Homenaje a Silvio Zavala II, julio-septiembre, 1989, pp. 7-19.

Herrejón Peredo, Carlos. “Aproximaciones a la obra de Silvio Zavala”, Relaciones, vol. XIV, núm. 56, 1993, pp. 131-135.

Las Casas, Bartolomé de, Historia de las Indias, México, Fondo de Cultura Económica, 1965.

León-Portilla, Miguel, "Corrientes o tendencias en la historiografía mexicana en los últimos veinticinco años", en Primer encuentro hispano-mexicano de historiadores, Madrid, Instituto Nacional de Ciencias de la EducaciónUniversidad Complutense, 1979, pp. 71-85.

Matute, Álvaro, "La historiografía positivista y su herencia", en Conrado Hernández, coord., Tendencias y corrientes de la historiografía mexicana del siglo $\mathrm{XX}$, Zamora, El Colegio de Michoacán-Universidad Nacional Autónoma de México-Instituto de Investigaciones Históricas, 2003, pp. 33-46.

Moctezuma Franco, Abraham, "El camino de la historia hacia su institucionalización", Historia y Grafía, núm. 25, 2005, pp. 45-78.

28 Sobre este tema ver Quijano, Las repúblicas de la Monarquía. 
O'Gorman, Edmundo. "Sobre la naturaleza bestial del indio americano", Thesis. Nueva Revista de Filosofía y Letras, núm. 1, 1979, pp. 7-20.

Pocock, John G. A., El momento maquiavélico. El pensamiento político florentino y la tradición republicana Atlántica, Madrid, Tecnos, 2002.

, Political Thought and History: Essays on Theory and Method, Cambridge, Cambridge University Press, 2009.

Quijano, Francisco, Las repúblicas de la Monarquía. Pensamiento republicano y constitucionalista, siglos XVI y XVII, México, Universidad Nacional Autónoma de México-Instituto de Investigaciones Históricas, 2017.

Skinner, Quentin, Los fundamentos del pensamiento político moderno, México, Fondo de Cultura Económica, 1985-1986 (dos volúmenes).

—, Visions of Politics, v. I, Regarding Method, Cambridge, Cambridge University Press, 2002.

Valero Pie, Aurelia, José Gaos en México: una biografía intelectual, 1938-1969, México, El Colegio de México, 2013.

Vázquez, Josefina Zoraida, "La historiografía mexicana en las décadas recientes", Revista de la Universidad, núm. 532, mayo, 1995, pp. 3-6.

Zavala, Silvio y Castelo, María, Fuentes para la historia del trabajo en Nueva España (1575-1805), México, Fondo de Cultura Económica, 1939-1946 (ocho volúmenes).

Zavala, Silvio, Ideario de Vasco de Quiroga, México, El Colegio de México, 1941.

— Los intereses privados en la Conquista de la Nueva España, México, Universidad Nacional Autónoma de México, 1964.

—- Recuerdo de Bartolomé de Las Casas, Guadalajara, Jalisco, Librería Font, 1966.

Las instituciones jurídicas en la Conquista de América, México, D.F., Porrúa, 1971.

—_ La encomienda indiana, México, Porrúa, 1973.

-, Servidumbre natural y libertad cristiana según los tratadistas españoles de los siglos XVI y XVII, México, Editorial Porrúa, 1975.

—_, La filosofía política en la Conquista de América, México, D.F., Fondo de Cultura Económica, 1977.

—_, Ensayos sobre la colonización española en América, México, D.F., Porrúa, 1978.

__ El servicio personal de los indios en el Perú, México, El Colegio de México, 1978-1980 (siete volúmenes).

, América en el espiritu francés del siglo XVIII, México, D.F., El Colegio Nacional, 1983.

—_. El servicio personal de los indios en la Nueva España, México, El Colegio de México-Centro de Estudios Históricos/ El Colegio Nacional, 1984, 1985, 1987, 1989, 1991, 1994, 1995 (siete volúmenes).

Por la senda hispana de la libertad, México, D.F., Fondo de Cultura Económica/Mapfre, 1993.

Zavala, Silvio, Peter Bakewell, y Dolores Gutiérrez Mills, “An Interview with Silvio Zavala", en The Hispanic American Historical Review, n. 62, 4, 1982, pp. 553568. 
Zermeño, Guillermo, La cultura moderna de la historia. Una aproximación teórica e historiográfica, México, El Colegio de México, 2002.

, "La historiografía en México: un balance (1940-2010)", Historia Mexicana, vol. LXII, núm. 4, abril-junio, 2013, pp. 1695-1742. 